
NOTICE

"This repon was prepared as an account of work sponsorcd by the United States Government. Neither the United States nor the United Siates Alumit Energy Commission, nor any of their employecs, nor any of their contrartots, subcontractors, or their employees, makes any warranty. express or implied, or assumes employees, makes any wir conty, express or inplied, or arsumes any legal lisbility or respondibility for the accuracy, completeness or usefulnes of any information, apparatus, produet or protess disclosed, of represents that its use would not infringe privatelyowned rights."

Printed in the United States of America Available from

National Technical Information Service

U.S. Department of Commerce 5285 Port Royal Road Springfield, Virginia 22151

Price: Printed Copy $\$$; Microfiche $\$ 2.25$

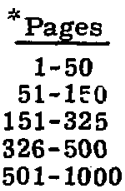

NTIS

Selling Price

$\$ 4.00$

$\$ 5.45$

$\$ 7.60$

$\$ 10.60$

$\$ 13.60$ 


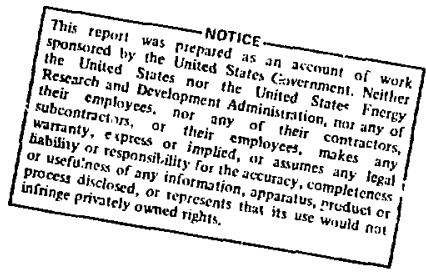

TID-4500, UC-34

Physics - General

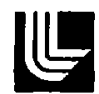

\section{LAWFENCE LNERMOPE LABOAATOFY}

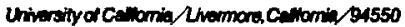

UCRL-51735

\section{PROJECTION-TUBE STUDIES \\ OF VOLTAGE-BREAKDOWN ARC DISCHARGES \\ FROM TUNGSTEN AND NIOBIUM WIRES IN VACUUM* \\ G. H. Miley ${ }^{\dagger}$}

MS. date: January 14, 1975

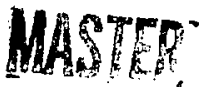

*This report is a written version of a talk presented at the 27th Annual Gaseous Electronics Conference, Houston, Texas, Oetober 1974.

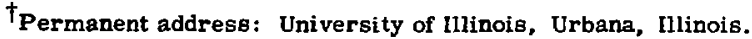




\title{
PROJECTION-TUBE STUDIES OF VOLTAGE-BREAKDOWN ARC DISCHARGES FROM TUNGSTEN AND NIOBIUM WIRES IN VACUUM
}

\begin{abstract}
The formation of "voltage breakdnwn" electric discharge arcs in vacuum between a wire cathode and a surrounding cylindrical anode has been studied with emphasis on behavior at the wire surface. Tungsten and niobium wires 2 to 10 mils in diameter were studied, using voltag':s up to $150 \mathrm{kV}$.

Conditioning of the wire by heating was found to have a strong effect on the behavior leading to breakdown. With unconditioned wire, very high prebreakdown currents were observed, and final breakdown occurred at low voltage. With parially conditioned wire (baked $1 \mathrm{hr}$ at $800^{\circ} \mathrm{C}$; , low pre-breakdown currents characteristic of field emission were observed. With further conditioning of the wire (baking overnight), no significant pre-breakdown emission occurred before fina! breakdown. Based on these observations, a breakdown correlation is propesed for design purposes that

involves three distinct regimes - initial, fleld emission, and ultimate - depending on prior conditioning of the surface.

With a projection tube used as the cylindrical anude, phosphorescent patterns on the tube caused by the discharges were recorded with a high-speed camera. and the corresponding areat of tn:- wire were studie $\rfloor$ with an electron microscope. Areas of the wire surface where pre-breakdown discharges occurred were found to be marked by micron-sized condensed droplets of aluminum and silicate, evidently transported there from the anode surface in plasma form during the discharge. Often these droplets were condensed on the ends of whiskers of wire material pulled up from the wire surface in a prebreakdown discharge. The final discharge appears to be a number of simultaneous ares, each originating in an urea of dense whisker formation on the wire surface.
\end{abstract}

\section{Introduction}

The objective of this researcin was to study the formation and discharge of arcs during voltage breakdown in vacuum. The experimental geometry involved a smail tungsten or niobium wire 2 to 10 mils in diameter, serving as the cathode, surrounded by a large cylindrical anode 2 to
4 in. in diameter. Th:s geometry made it possible to achieve surface fields of the order of $1 \mathrm{MV} / \mathrm{cm}$ on the wire cathode with modest power supply voltages $(<100 \mathrm{kV})$. The apparatus is shown in Fig. 1 with the vacuum-chamber bell jar raised. 


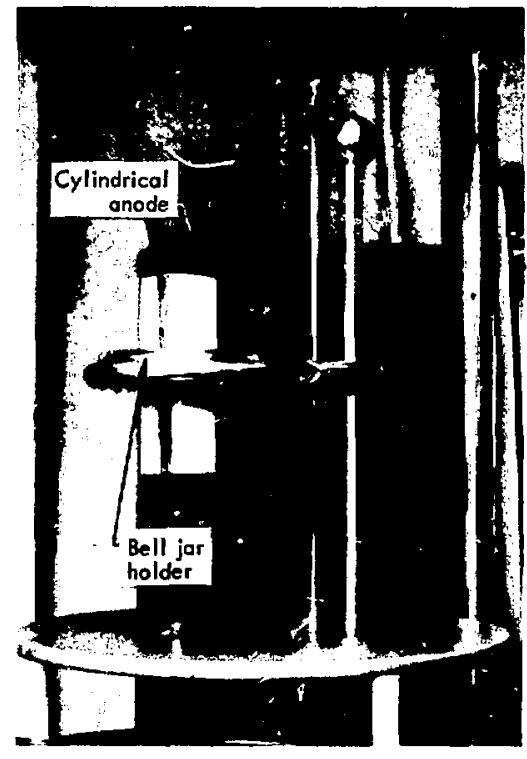

These studies were motivated by two applications important to fusion reactor development: Breakdown from grid surfaces represents a limiting ferture in direct conversion devices intended to collect and electrostaticaliy convert fusion-ion energy to high-voliage do electrical output. Also, a similar critical problem occurs at the high-voltage extraction grids in high-energy ion sources which are under development for injection into fusion plasmas.

Fig. 1. The voltage breakdown apparatus used in this study.

\section{Voltage-Current Characteristics}

While breakrdown from wires has been studied frr many years te.g., Ahearn and Chambers in the 19:30's). no general breakdown critrrian has bera published.' To investigate sunu if the basir eharacteristics of breakdıwn, nur initial runs were carrired ull with stainless sle.e? anodes. Provision was made for conditioning the wir! breing studie.rl. It was possible to resistively heat the wire while visltage was applted, and baking of the wre in situ (t, $e^{2}$, at hard vacuum) was exteusively used fur andituoning.

1. An extersive revelu al earlier work, breakdowi: theors, atut destgu correlations was prestet di lts an earlier report by the atuti:ur (LCR1-51482, ver. 114731 .
Some typical current-voltage plots are shown in Fig. 2 for a 7 -mil tungsten wire. Three regimes of operation are illustrated in Fig. 2, all related to prior conditioning of the wire. In the initial regime, i.e., before conditioning of the wire, very high pre-breakdown currents were observed, and breakdown occurred at low voltage. After the wire was partially conditioned by baking it at $\sim 800^{\circ} \mathrm{C}$ for an hour, low pre-breakdown currents were observed which are characteristic of field emission; hence this is termed the field emission regime. An ultimate regime could be obtained by baking the wire overnight where no field emission was observed prior to breakdown. 


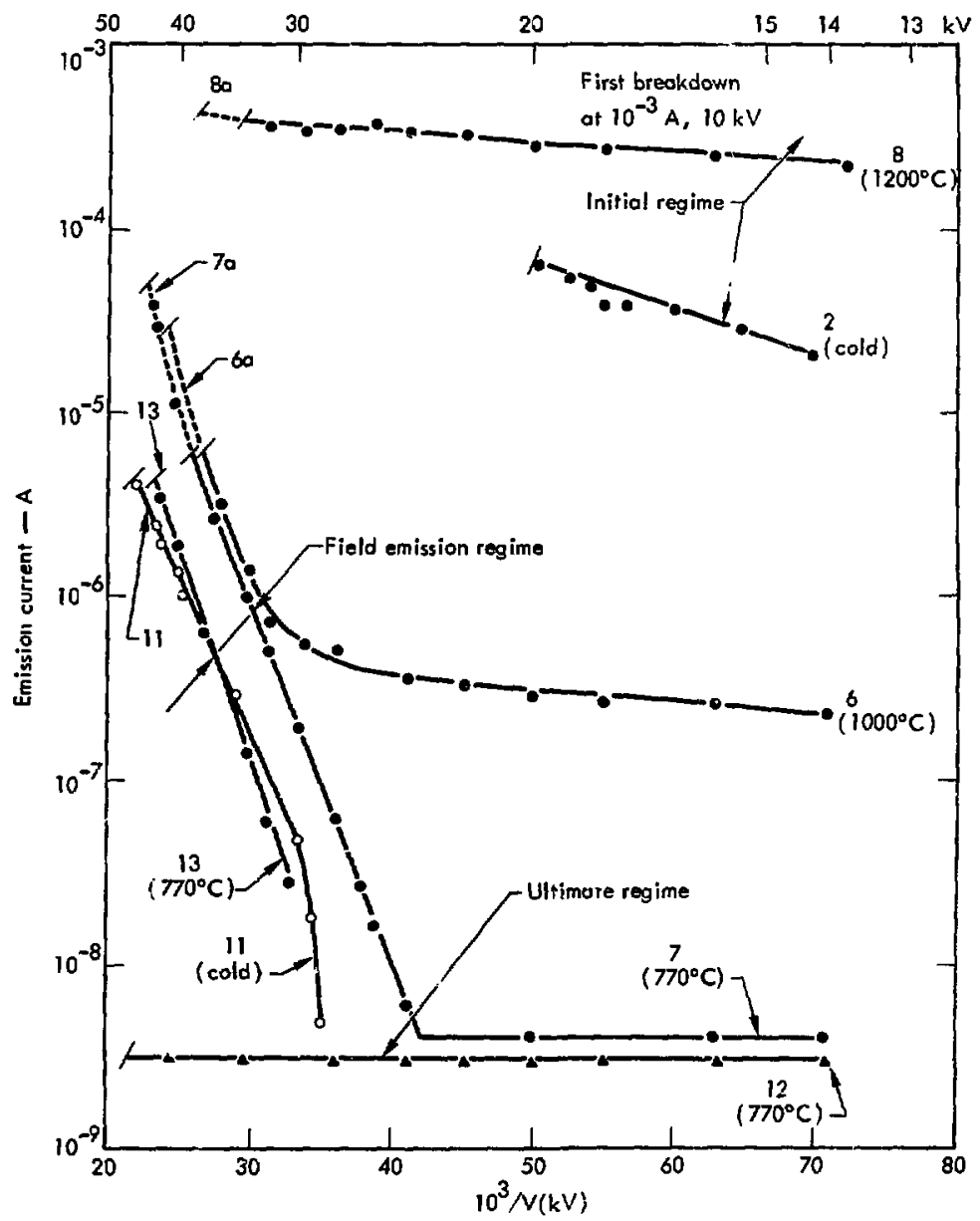

Fig. 2. Typical plot of emission current vs voltage for a 7-mil-diam tungsten wire after various conditioning processes.

In addition, it will be noted from

Fig. 2 (runs 12, 6, and 8) that the final voltage breakdown point remained fixed independent of wire temperature during the run, even when thermionic prebreakdown emission currents from the hot wire were much larger than the prebreakdown currents from the cold wire. 


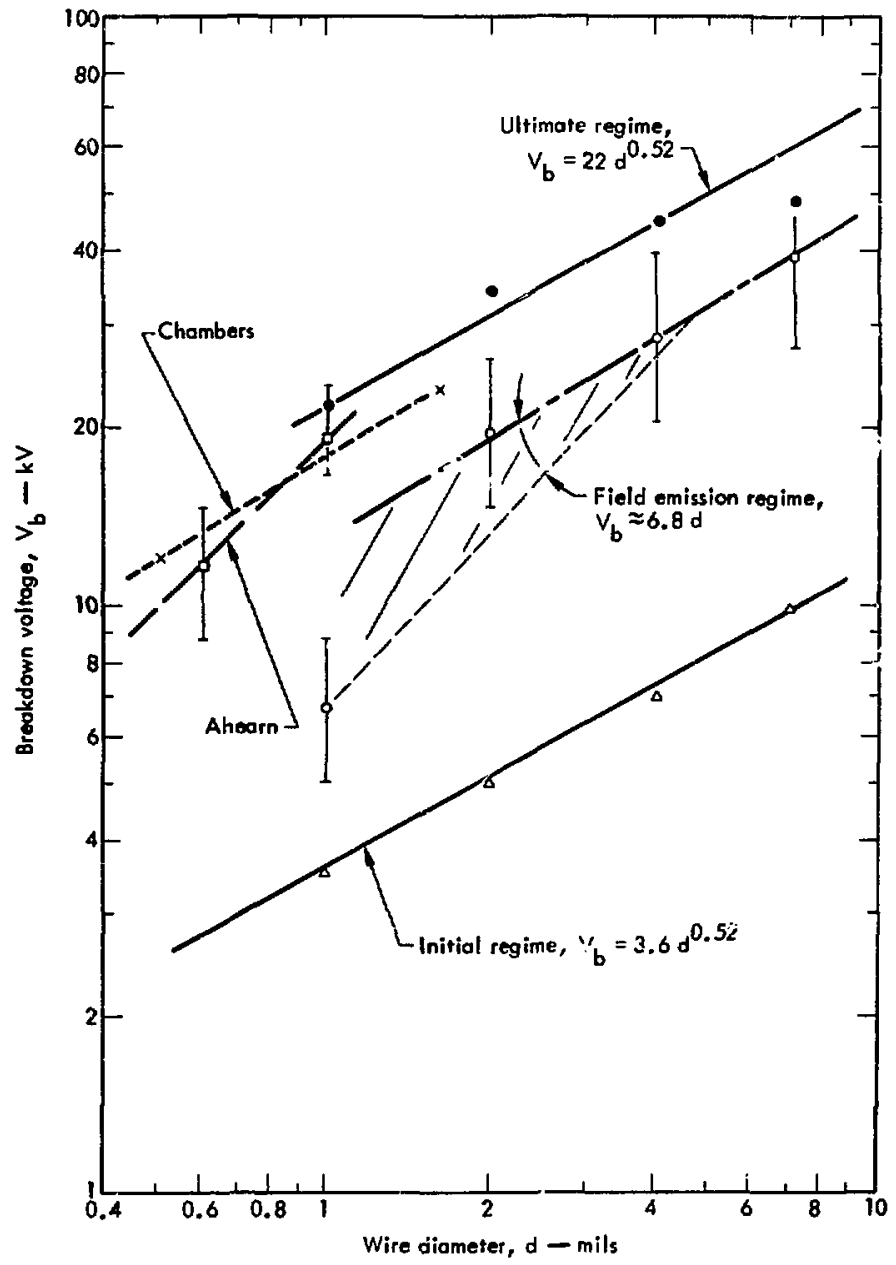

Fig. 3. Voltage breakdown correlation as a function of wire diameter.

These data are consistent with the following physical interpretation. Dirty surfaces prior to conditioning cause lacge pre-breakdown currents and er- ratic breakdown behavior. Arcs can be initiated by clumps of material or gases leaving the surface. After partial conditionirig, only !cw pre-breakdown cur- 
rents are observed until the voltage becomes large enough to raise surface protrusions or whiskers. Due to the ?arge length/diameter ratio of such a whisker, the electric field at the tip of the whisker is enhanced by a factor of $10^{3}$ of more, causing characteristic field emission. Breakdown becomes more predictable because these emission currents initiate arcs by, for example, melting whiskers. So:ne scatter still occurs, however, due to the random nature of the number, size, and shape of the whiskers, In the ultimate regime, the surface is free of whisker 3 , at least up to the moment of arc initiation.

These concepts are illustrated again in the plot of breakdown voltage vs wire diameter in Fig. 3. While all three regimes tend toward a correlation of the form $v_{b} \propto d^{1 / 2}$, the actual breakdown mechanisms are different in each. This is not surprising since well-known theories such as the Cranberg clump-modeI, various whisker-emission theories, etc. all suggest similar correlations.

\section{Projection Tube Studies}

To study the arc formation more closely, a projection tube ${ }^{2}$ was substi tuted for the outer cylinder (anode). As shown in Fig. 4, this is simply a glass tube coated on the inside with a phosphor. Electron surrents produce a bright glow on the phosphor which is visible through the glass. A thin aluminum coating on the inside (wire side) of the phosphor provides electrical contact.

Projection tubes have been used by several workers prior to this study, but their tubes were not :ugged enough to study high currents or actual arc breakdown. The present design was worked

2. !. Brodie and L. Weissman, Vacuum 14. 299 (1964).

Fig. 4. Glass anode with internal phosphor coating (the "projection tube").

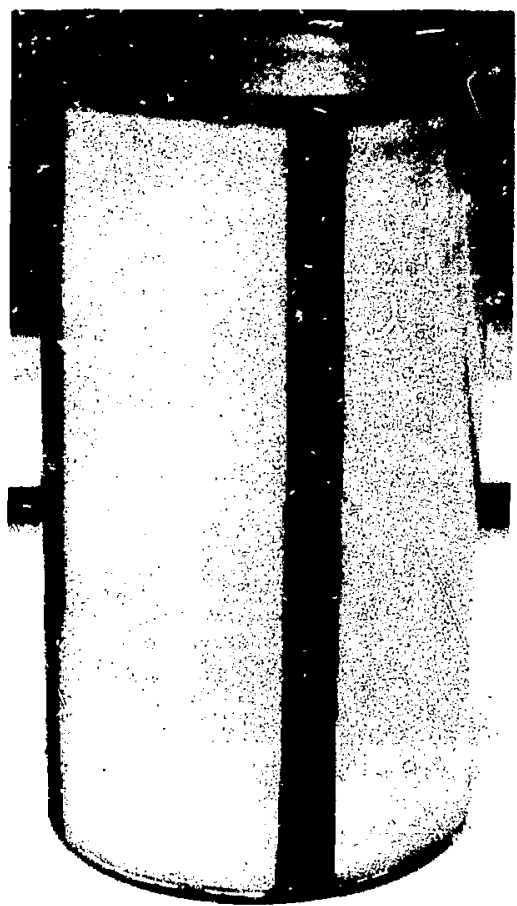


out to allow these investigations, and good success was obtained although damage to the tudes did eventually occur aster extensive arcing.

Both still and high-speed sequential photography were used to record the dynamirs of spot frowth and breakdown. A typical variation of the spots with increasing voltage is shown in Fig. 5 . While the current-voltage characteristics remained fairly snooth as shown eariier. the size and location of the currentcarrying spots undeiwent constart variations. The birth or death of spots, even at constant voltage, was frequently observed, and this was accentuated with changing voltage.

It was found, as illustrated in Fig. 6, that the emission current was closely correlated with the spot dersity for a wide range of conditions and wirer. The spot density appears, however, to :ncrease somewhat faster than predicted by a inear relation with current. This is probably to be expected in light of the statistical variation in the shape and current-carrying capacity of individual whiskers.

T:zese observations are in general agreement with the earlier picture of a dynamic "eruption" of whiskers from the surface under the influence of the applied field. The spot size and shape observed on the screen can be used to make an estimate of the whisker size, The highspeed sequential photographs allow a gross observation of the final breakdown, bint the resolution is poor hecause of the relatively slow time response of the phosphor. Perhaps the most striking observation is that a number of ares appear to erupt simultaneously along the wire rather than at a single spot. Thus it is thrught that debris and shock effects proliferate the arcing along the surface.

\section{Surface Studies}

The projection tube made it possible to locate the precise emission sites causing the pre-breakdown currents. The section of the wire leading to bright spots was sectioned and studied unde: a scanning electron microscope. In ronse instances a surface configuration such as shown in Fig. 7 was found. The spherical object in this photo is roughly $6 \mu \mathrm{m}$ in diameter, and it is on whisker about 2 by $10 \mu \mathrm{m}$ that project - from the wire. Microprobe analysis shows that the spherical ball is mainly composed of aluminum and silicate from the phosphor and electrical coating on the projection tube. The whisker supporting the ball is mainly material from the wire, with a thin outer coating of materials from the projection tube. This suggests that the pre-breakdown currents involved a low density plasma composed of electrode material. Such vapor formation has been observed in other studies. ${ }^{3}$ Due to its low melting point, aluminum dominated the plasms, and it was gradually condensed out on the whisker tip.

Studies were made of damaged areas on the surface after arc breakdown

3. D. Davies and M. A. Bior.di, J. Appl. Phys. 41, 88 (1970). 

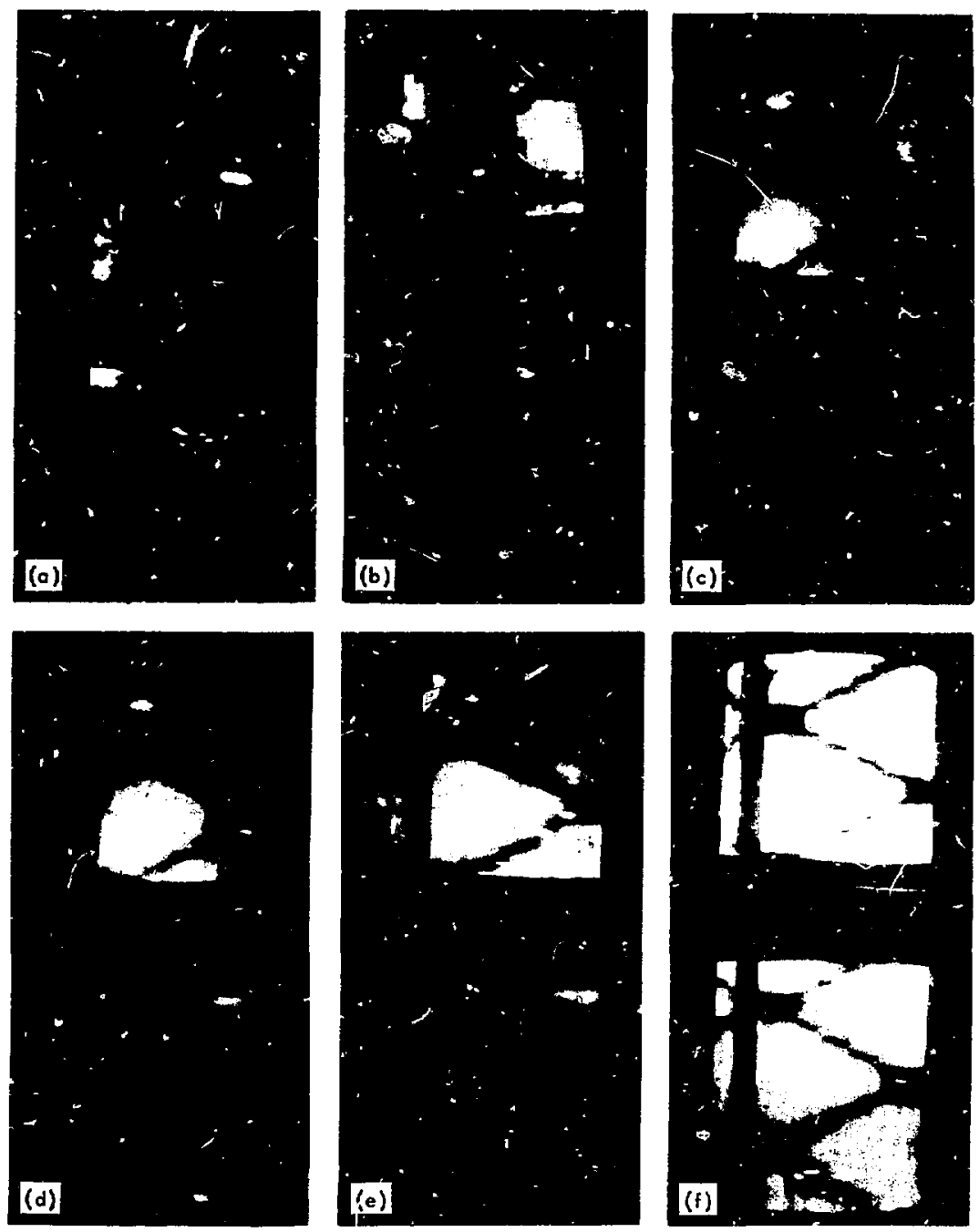

Fig. 5. Photograpins of sequence of spots observed on the projection tube wi lincrasing voltage. 


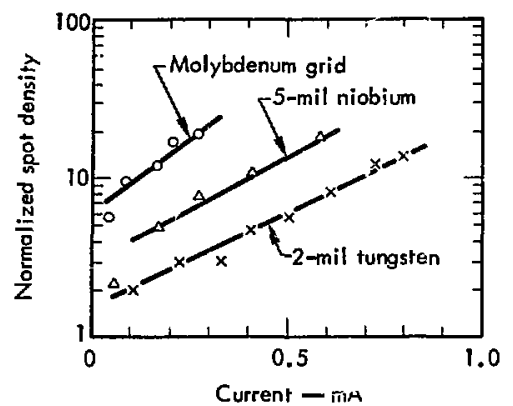

Fig. 6. Density of spots observed on the projection tube vs pre-breakdown current.

scurred, and the results suggested that the final arc originated in an area of ciense whisker formation. For example. Fig. 8 shows some ball/whisker formations around the edge of an arc-created

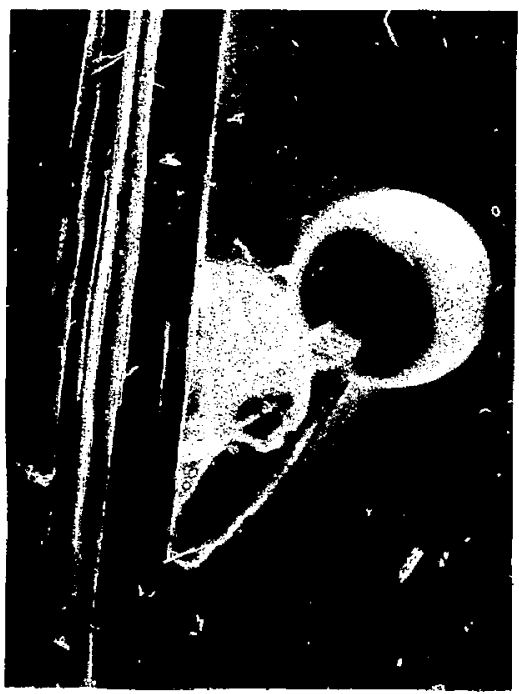

Fig. 7. Electron microscope photograph of a whisker on the surface of a niobium wire. Microprobe analysis indicates that the ball on the whisker is mairly aluminum $(\sim 5300 \times)$. crater that were partly melted by the breakdown but remain clearly visible.

One consequence of these observations is that the deposition of materials on whisker surfaces as well as the damage to their crystalli estructure during erection are now thought to affect the work function of this localized area. Thus large emission currents can be obtained with relatively small field enhancement, i.e., length, liameter ratios. Such a mechanism woul 1 be consistent with earlier observations by Brodie, ${ }^{4}$ who found that minute quantities of material evaporated from adjacent thermionic eathodes "activated" the tips of existing whiskers on nickel electrodes, causing enhanced field emission.

4. 1. Brodie, J. Vac. Sci. Technol. 2 249 (1965).

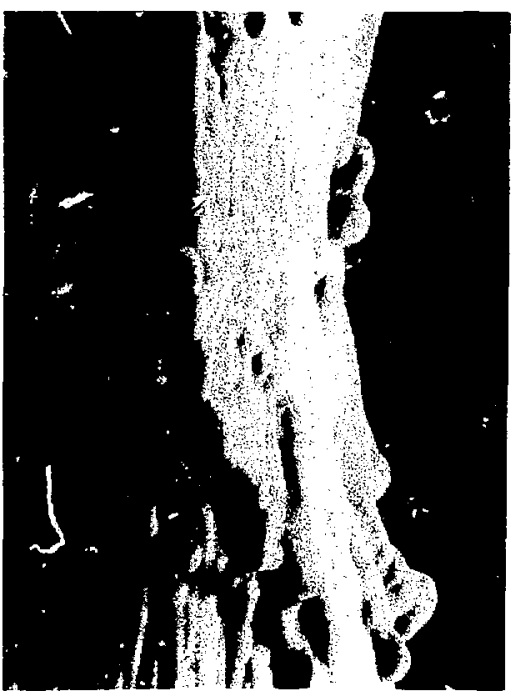

Fig. B. Electron microscope photograph of a damaged area with whisker/ ball formations near the edge of the crater region $(\sim 4400 \times)$. 


\section{Conclusions}

This work has demonstrated that the projection tube technique provides a valuable method for studies of prebreakdown and breakdown arcing in vacuum. In addition to providing information about arc dynamics, the spots observec made it possible to accurately locate wire sectiors for subsequent studies with a scanning electron microscope.

The observations add important evidence for a dynamic model of whisker formation :y field erections, followed by pre-breakdown emission involving transport, via low-density plasma, of anode material to the whisker. This emission eventually grows into a full arc while simultaneously propagating to other sites along the surface. For design purposes, a voltage breakdown correlation is proposed that involves three distinct regimes of breakdown - initial, field emission, and ultimate - depending on prior conditioning of the surface.

Work is now in progress to employ the projection tube technique to study voltage breakdown from wire surfaces blistered by helium bombardment. Results from these studies will be reported in the near future.

\section{Acknowledgmenta}

The continuing support and advice in this work by R. Moir is appreciated.

J. Kinney, W. Gould, and C. Hansen were instrumentai in setting up the apparatus and carrying out the experiments.

WEC/rt/la 